\title{
Fast tunable ultra-violet laser source for confocal fura-2AM imaging
}

\author{
E Esposito, J Harris and G McConnell \\ Centre for Biophotonics, Strathclyde Institute of Pharmacy and Biomedical Sciences, \\ University of Strathclyde, 27 Taylor Street, Glasgow, G4 0NR, United Kingdom
}

Email: g.mcconnell@strath.ac.uk

\begin{abstract}
We report upon a novel wavelength-flexible laser source for three-dimensional ultraviolet imaging. Based on supercontinuum generation in photonic crystal fiber, the resultant broadband source extended from $\lambda=331 \mathrm{~nm}$ into the visible region of the spectrum. Using an electronically-controlled filter wheel and filter set with a response time of approximately $50 \mathrm{~ms}$, rapid wavelength selection was performed. The described scheme is capable of exciting the current range of ultra-violet-excited fluorophores and the simple and rapid wavelength control also provides a new approach for fast ratiometric imaging of Fura-2AM, facilitating an easy method of performing quantitative intracellular calcium concentration measurements.
\end{abstract}

\section{Keywords}

42.62.Be Biological and medical applications, 42.65.Ky Frequency conversion, 87.64.Tt Confocal microscopy

\section{Introduction}

Confocal laser scanning microscopy (CLSM) is of growing importance for a wide range of applications in the life sciences. The high-resolution optical cross-sectional images enable the three-dimensional reconstruction of fluorescent samples. The use of light, rather than histological sectioning methods, provides a minimally invasive method of visualising cells and can be used to study dynamic cellular processes [1]. To maximise the efficiency of the process, the excitation source should be chosen carefully in order to minimise photo-damage. Typically, the fluorophores used in CLSM require a laser source absorb either ultraviolet or visible wavelengths. A supercontinuum laser source has been shown previously to be capable of visiblewavelength CLSM but this did not cover ultraviolet wavelengths [2]. Recently, supercontinuum generation at these shorter wavelengths was reported using photonic crystal fiber [3]. Extending the application of shorter-wavelength supercontinuum generation for CLSM simplifies the technique and opens up a wider range of applications in the life sciences and beyond.

For example, at present, $\mathrm{Ca} 2+$ activity is typically measured using the Fura range of dyes. These mostly depend upon a shift in emission wavelength to accurately measure $\mathrm{Ca} 2+$ activity. In order to accurately measure this change, the detector employed must have a linear response across the wavelength range. In practise, this is very difficult to achieve and hence alternatives are desirable. Fura-2AM operates slightly differently insofar as the excitation wavelength is varied in order to measure the $\mathrm{Ca} 2+$ levels through a change in fluorescence at a fixed wavelength. 
Fura-2AM is routinely used in widefield ratiometric imaging of live cells to image $\mathrm{Ca}^{2+}$ activity $[4,5,6]$. The ratio of the resultant fluorescence is subsequently used to quantify and measure the $\mathrm{Ca}^{2+}$ level within the cell [4]. In widefield imaging, the wavelengths are usually supplied by a high-pressure arc lamp and a rapidly controlled monochromator, for example [7]. However, the options for performing ratiometric CLSM with Fura-2AM are limited, due to the lack of laser sources with the necessary dual emission wavelength. Most commonly, the $\lambda=351 \mathrm{~nm}$ line of a $\mathrm{Kr} / \mathrm{Ar}$ laser source provides excitation but does not provide the wavelength tenability required for efficient CLSM of Fura-2AM. Further to Fura-2AM imaging, many routinely used fluorophores such as Indo-1 and Alexa 350 would benefit from a tunable ultra-violet laser source [8], as would applications in ultra-violet photolysis of caged molecules [9].

To overcome this technology limitation, we have developed a wavelength-flexible ultra-violet laser source for CLSM. Using nonlinear optical processes in photonic crystal fiber, we have engineered an ultra-violet supercontinuum laser source extending from $\lambda=331 \mathrm{~nm}$ into the visible range of the spectrum. This source provides the wavelength range necessary to excite the entire current range of commercially available ultra-violet absorbing fluorophores. Furthermore, our application of rapid wavelength switching methods provides a light source that is capable of ratiometric $\mathrm{Ca}^{2+}$ microscopy using Fura-2AM.

\section{Experiment}

The experimental configuration is shown in Figure 1. A commercial Nd:YLF laser (Biolight, Coherent) was used as the platform source to pump the photonic crystal fiber. With an emission wavelength of $\lambda=1047 \mathrm{~nm}$ and a spectral FWHM of $\Delta \lambda=$ $6 \mathrm{~nm}$, the horizontally-polarised Nd3+:YLF laser delivered pulses of approximately $400 \mathrm{fs}$ duration at a repetition rate of $120 \mathrm{MHz}$ and an average power of $700 \mathrm{~mW}$ in a single-mode beam. This corresponded to a peak power of over $15 \mathrm{~kW}$. Confirmation of mode-locked operation was performed over the course of the experiment by directing $<4 \%$ of the beam towards an optical spectrum analyser (USB 2000, Ocean Optics bV) and monitoring the spectral FWHM. The remaining $96 \%$ of the output power from the platform laser was used in the experiment. Following steering from a highly-reflecting plane mirror, the beam was propagated through a $30 \mathrm{~dB}$ Faraday isolator to minimise Fabry-Perot reflections that otherwise disrupted pulse generation in the laser resonator via feedback. The radiation transmitted by the Faraday isolator was then propagated through a half-wave plate designed for operation at $\lambda=1047$ $\mathrm{nm}$ to control and rectify polarisation offset. This beam was then focussed using a miniature spherical lens with a focal length of $f=3.1 \mathrm{~mm}$ (Thorlabs) into a $20 \mathrm{~cm}$ long section of photonic crystal fiber (NL-2.0-745, Crystal Fiber). The coupling efficiency was approximately $40 \%$. The light transmitted by the PCF was then collimated using a second miniature lens element with a focal length of $8 \mathrm{~mm}$ (Thorlabs). The infrared radiation from the pump source was blocked using a high-quality short-wavelength pass interference filter (400SWP, Chroma), allowing only the transmission of wavelengths shorter than $\lambda=400 \mathrm{~nm}$. These wavelengths served as the ultra-violet source for confocal excitation. The ultra-violet source was subsequently coupled into a commercial laser scanning microscope (Bio-Rad MRC1000) designed for ultraviolet operation before reaching an inverted microscope (Diaphot 200, Nikon) with a $40 \mathrm{x} / 1.3$ oil-immersion ultra-violet corrected objective lens. The fluorescence signal was collected by the same objective lens and directed towards a photomultiplier tube within the scan-head and the resultant signal used to construct a contrast image.

Wavelength discrimination from the spectrally broad ultra-violet laser was performed using a spectrophotometer comprising an electronically controlled filter wheel with six 
changeable optical bandpass filters (Cairn). The spectrophotometer could be operated in a time/synch-pulse mode and therefore provided the possibility of true synchronism with the laser scanning microscope. The filter position could be changed at rates of up to $96 \mathrm{~Hz}$, but the system was not used at maximum speed due to the image acquisition rate of the laser scanning microscope of approximately $1 \mathrm{~Hz}$. However, the light source and electronically controlled filter wheel offers the possibility to perform fast confocal microscopy which has implications for studying dynamic processes in living cells, such as performing ratiometric $\mathrm{Ca}^{2+}$ imaging.

\section{Results}

The un-filtered spectrum transmitted by the PCF is shown in figure 2, measured using a fiber-coupled spectrometer with a minimum wavelength response of $\lambda=330$ $\mathrm{nm}$. It is entirely possible that wavelengths shorter than the response of the spectrometer were generated but not detected. The average power across the spectral range after the $400 \mathrm{~nm}$ SWP filter was measured to be $30 \mathrm{~mW}$. The measured spectrum revealed some very minor intensity fluctuations on the spectral structure of the transmitted light but these were found to be significantly less than $\pm 0.25 \%$ (rms). This compared well with the traditional gas-based lasers typically applied for ultra-violet confocal microscopy [1].

A range of samples were imaged using the described system. Firstly, the resolution of the laser scanning microscope with the ultra-violet supercontinuum source was evaluated using flow cytometry alignment beads of $2.5 \mu \mathrm{m}$ diameter (Alignflow A7305, Invitrogen). These beads are used in the calibration and focusing of flow cytometers, but their excitation wavelength range of $\Delta \lambda=350-370 \mathrm{~nm}$ provided a simple method of measuring the lateral and axial resolution. To this end, a $\lambda=360 \pm 10 \mathrm{~nm}$ filter was used to isolate a narrow spectral range from the ultra-violet supercontinuum to excite the sample. The emission wavelength of the beads was specified between $\Delta \lambda=400-470 \mathrm{~nm}$. To discriminate fluorescence from backscattered excitation light, a long wave pass filter allowing only wavelengths longer than $\lambda=390 \mathrm{~nm}$ to reach the photomultiplier tube detector was used.

Typical fluorescence and transmission cross-sectional images of ultra-violet-excited beads are shown in figure 3 . The images were captured at an acquisition rate of approximately $1 \mathrm{~Hz}$, at a box size of $512^{2}$ pixels. A Kalman averaging filter was applied over $n=4$ images to improve the signal to noise ratio. From analysis of the resultant images (Metamorph, Universal Imaging), the lateral and axial resolution were close to the diffraction limit. The lateral and axial resolutions are presented in table 1. Given the high lateral and axial resolution, the system was then employed for cellular imaging.

Chinese hamster ovary $(\mathrm{CHO})$ cells were loaded with $1 \mu \mathrm{M}$ of Fura 2-AM (Invitrogen) and the cells were allowed to adhere to a glass coverslip placed on the microscope stage. An optical bandpass interference filter with a peak transmission wavelength of $\lambda=340 \pm 25 \mathrm{~nm}$ (Comar) was placed prior to the scan-head to discriminate the useful excitation radiation for the fluorescent indicator. Attenutation of the average power was performed by a low-power neutral density filter. The average power at the microscope after the objective lens for this spectral range was measured to be 0.7 $\mathrm{mW}$ and this light served as the excitation for the sample. Figure 4(a) and (b) show typical fluorescence and transmission cross-sectional images of the $\mathrm{CHO}$ cells loaded with Fura-2AM. As with the alignment beads, the cells were imaged at an acquisition rate of roughly $1 \mathrm{~Hz}$, with a box size of $512^{2}$ pixels. Furthermore, a Kalman averaging filter was applied over $n=6$ sequential scans. As a control test, $\mathrm{CHO}$ cells with no Fura-2AM were also imaged and the fluorescence signal 
compared with the dye-loaded cells. As shown in figure 4(c), the unstained cells produced virtually no fluorescence in comparison with the stained cells under the same conditions. It is critical to note that continued imaging of the $\mathrm{CHO}$ cells over an hour-long period ( $\mathrm{n}=6$ individual samples) did not cause morphological change to the cell, i.e. membrane blebbing or shape change, that is generally indicative of lightinduced cell damage. This is of particular importance for ultra-violet confocal laser scanning microscopy, where the application of shorter wavelength radiation may lead to cell damage.

The laser scanning microscope used in this investigation had a maximum acquisition rate of approximately 4 frames per second. This was not sufficiently rapid to study dynamic intracellular $\mathrm{Ca}^{2+}$ events, but the light source described was easily wavelength switchable and capable of providing variable spectral bandwidth excitation at speeds of greater than $100 \mathrm{~ms}$. For increased speed, alternative spectral selection methods such as digital micro-mirror devices provide a flexible, if rather more expensive option [10].

\section{Conclusion}

In conclusion, we have demonstrated the application of an ultra-violet supercontinuum laser source for high-resolution CLSM of live cells loaded with the $\mathrm{Ca}^{2+}$ indicator Fura-2AM. The broad spectrum available from the ultra-violet supercontinuum provides a wavelength flexible source capable of exciting a wide range of fluorophores and fluorescent media. Employing a rapid spectrophotometer to extract narrowband wavelength ranges from the supercontinuum for excitation of Fura-2AM offers the possibility for confocal ratiometric $\mathrm{Ca}^{2+}$ measurements and we will investigate this in due course using a fast CLSM system.

\section{Acknowledgements}

The authors wish to acknowledge financial support from the Royal Society and the Royal Society of Edinburgh. 


\section{Figures}

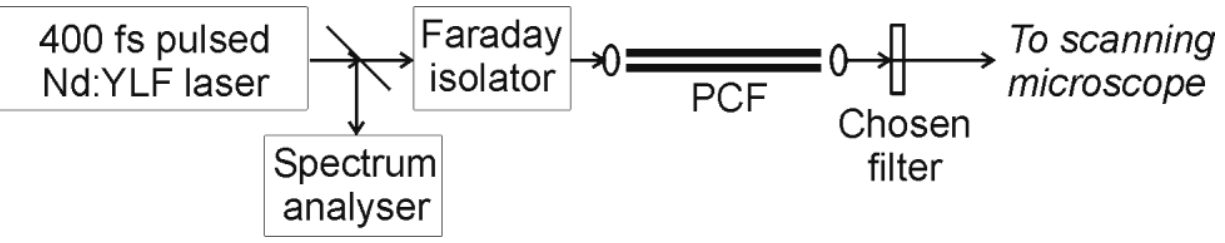

Figure 1. Experimental configuration to generate the ultra-violet supercontinuum and subsequent application for confocal laser scanning microscopy. 


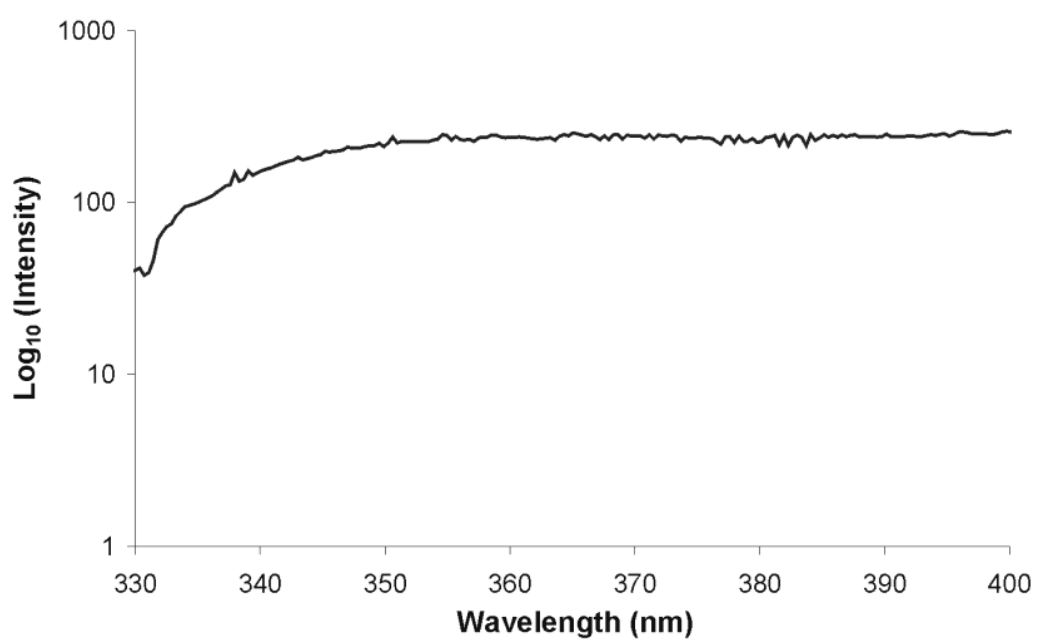

Figure 2. Typical unfiltered ultra-violet supercontinuum transmitted through the PCF. This corresponds to an average power of approximately $30 \mathrm{~mW}$ emitted through the PCF. 


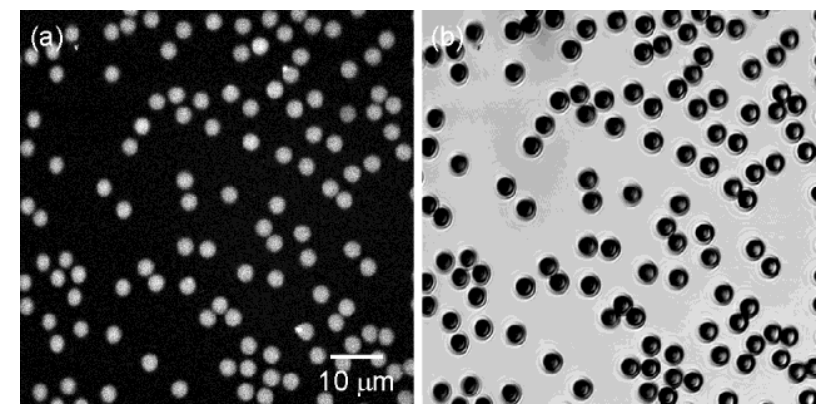

Figure 3(a) Fluorescence and (b) transmission confocal images of Alignflow ultraviolet excited fluorescent beads of $2.5 \mu \mathrm{m}$ diameter. An optical zoom factor of 3 was employed for additional magnification. 

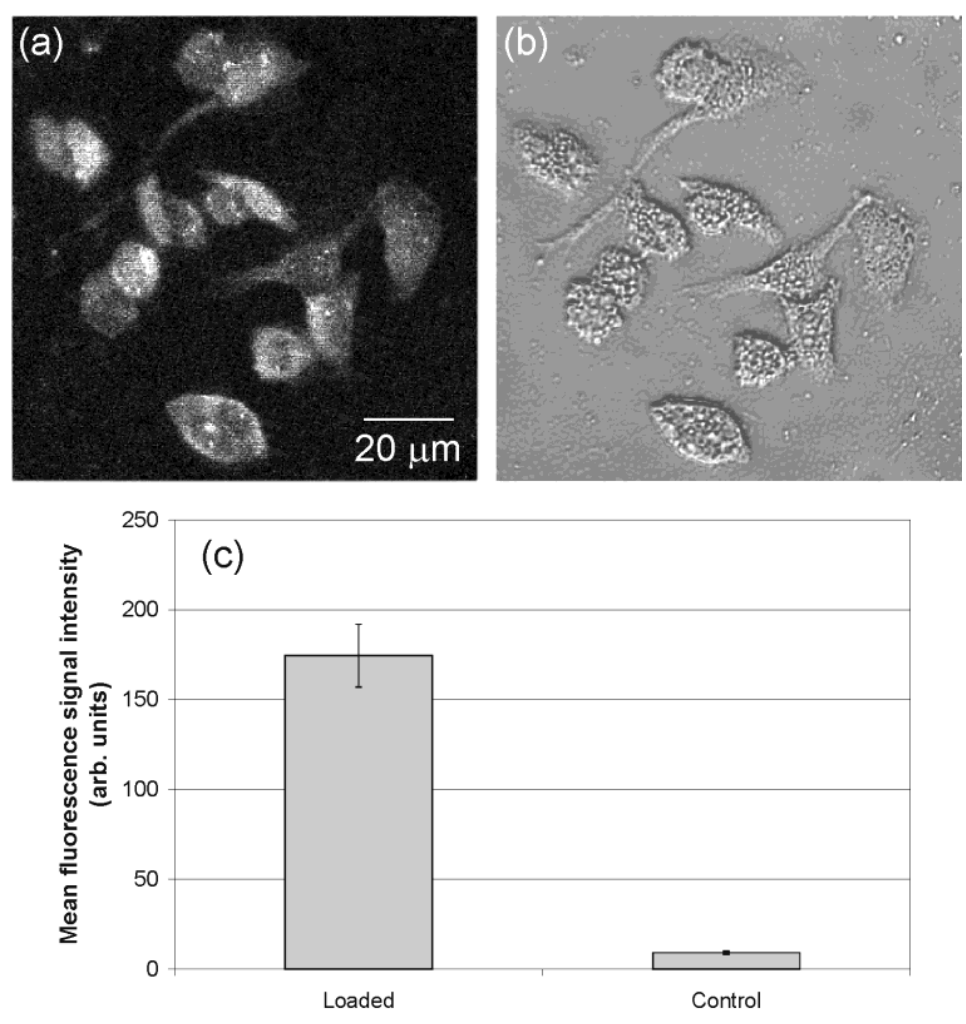

Figure 4(a) Fluorescence and (b) transmission confocal images of $\mathrm{CHO}$ cells loaded with $1 \mu \mathrm{M}$ solution of the fluorescent $\mathrm{Ca}^{2+}$ indicator Fura-2AM. Figure 4(c) shows the levels of fluorescence from the loaded cells in comparison with the control cells with no applied fluorophore. 
Table 1. Theoretical and measured resolution

\begin{tabular}{|l|c|c|}
\hline & Measured & Theoretical \\
\hline Lateral Resolution $(\mathrm{nm})$ & $183 \pm 0.05$ & 164 \\
\hline Axial Resolution $(\mathrm{nm})$ & $426 \pm 0.07$ & 414 \\
\hline
\end{tabular}

Table 1. Comparison of the theoretical and measured lateral and axial resolutions for a given excitation wavelength of $\lambda=350 \mathrm{~nm}$ [11]. 


\section{References}

[1] J.M. Girkin and G. McConnell, Micro. Res. Tech. 67 (2005) 8-14.

[2] G. McConnell, Opt. Express 12 (2004) 2844-2851.

[3] J.H.V. Price, T.M. Monro, K. Furusawa, W. Belardi, J.C. Baggett, S. Coyle, C. Netti, J.J. Baumberg, R. Paschotta, D.J. Richardson, App. Phys. B. 77 (2003) 291298.

[4] H. Hayashi, H. Miyata, J Pharmacol Toxicol Methods. 31 (1994) 1-10.

[5] W.G. Wier, L.A. Blatter, Cell. Calcium.12 (1991) 241-54.

[6] R.A. Hirst, C. Harrison, K. Hirota, D.G. Lambert, Methods Mol Biol. 114 (1999) 3139.

[7] I. Maric, J.L. Barker, Methods 21 (2000) 335-347.

[8] W.G. Telford, Cytometry A 61 (2004) 9-17.

[9] S.S. Wang and G.J. Augustine, Neuron 15 (1995) 755-60.

[10] G. McConnell, S. Poland and J.M. Girkin, Rev. Sci. Instrum. 77 (2006) $013702-$ 01370.

[11] J.B. Pawley, Handbook of Biological Confocal Microscopy $2^{\text {nd }}$ edn, New York: Plenum, 1996. 